\title{
PW03-009 - Genetics of PFAPA syndrome
}

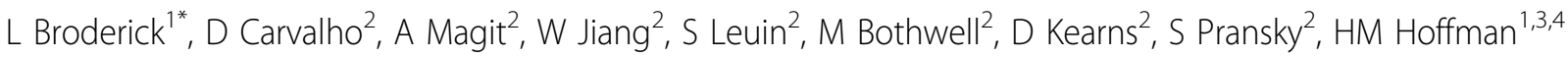 \\ From 7th Congress of International Society of Systemic Auto-Inflammatory Diseases (ISSAID) \\ Lausanne, Switerland. 22-26 May 2013
}

\section{Introduction}

Periodic Fever, Aphthous stomatitis, Pharyngitis and Adenitis (PFAPA) syndrome is an autoinflammatory disorder of childhood and little is known about the underlying etiology. While mutations involving the IL-1 pathway have been identified in other recurrent fever disorders, including TNF-receptor associated periodic syndrome (TRAPS) and cryopyrin-associated periodic syndrome (CAPS), PFAPA syndrome is not traditionally considered to be a hereditary fever disorder.

\section{Objectives}

To evaluate pediatric patients with PFAPA for family histories suggestive of immune dysregulation and to correlate inheritability with immune phenotype.

\section{Methods}

Patient data and detailed family histories were collected for over 170 children with recurrent fevers including 70 patients with PFAPA to create a prospective cohort over a 4-year period. DNA was isolated from blood or tonsillar tissue from recurrent fever patients, and NLRP3 and TNFRSF1A were sequenced. Quantitative real time PCR was used to evaluate $I L-36$ transcripts in tonsils.

\section{Results}

Our cohort reflects the diversity of San Diego, without predilection for any specific ethnic background. Family histories revealed $21 \%$ of patients have a first degree relative with recurrent fevers and $12 \%$ with tonsillitis in childhood, with only $1.4 \%$ reporting a history of recurrent infections. We have identified over 30 families with 2-8 affected members. These patients do not possess mutations commonly seen in other autoinflammatory disorders such as CAPS or TRAPS, suggesting that a novel gene may be involved. Upregulation of IL-36
mRNA expression in tonsils identifies the IL-1 family member IL-36 as a candidate gene.

\section{Conclusion}

A substantial portion of our families with PFAPA report childhood histories of recurrent fevers that resolved either spontaneously or with tonsillectomy, indicating a possible dominantly inherited trait that impacts the developing immune system, including the tonsils.

\section{Disclosure of interest}

L. Broderick: None declared, D. Carvalho: None declared, A. Magit: None declared, W. Jiang: None declared, S. Leuin: None declared, M. Bothwell: None declared, D. Kearns: None declared, S. Pransky: None declared, H. Hoffman Consultant for: Regeneron, Novartis, and Sobi.

\section{Authors' details}

${ }^{1}$ Medicine, University of California, San Diego, USA. ${ }^{2}$ Surgery, University of California, San Diego, USA. 'udwig Institute of Cancer Research, San Diego, USA. ${ }^{4}$ Pediatrics, University of California, San Diego, San Diego, USA.

Published: 8 November 2013

doi:10.1186/1546-0096-11-S1-A235

Cite this article as: Broderick et al:: PW03-009 - Genetics of PFAPA syndrome. Pediatric Rheumatology 2013 11(Suppl 1):A235.

${ }^{1}$ Medicine, University of California, San Diego, USA

Full list of author information is available at the end of the article 